\title{
Los pinzones de Darwin y otras aves de Galápagos: lo que Charles Darwin vio y no percibió
}

\author{
Tjitte de Vries \\ Escuela de Ciencias Biológicas de la Pontificia Universidad Católica del Ecuador. \\ tdevries@puce.edu.ec
}

En los primeros días de su estadía de cinco semanas en Galápagos, Charles Darwin no se dio cuenta que habían diferencias importantes en los animales provenientes de las diferentes islas. Por tal motivo, sus colecciones de las dos primeras islas que visitó, San Cristóbal (Chatham) y Floreana (Charles), fueron mezcladas. En algunos casos fueron rotuladas simplemente como provenientes de "Galápagos".

Cuando en Floreana el Gobernador de las Islas le contó que él podía distinguir a las tortugas gigantes de cada isla por su forma y tamaño, Charles Darwin reflexionó y dijo: "I must suspect they are only varieties" (Yo sospecho que son solamente variedades). En Galápagos, Charles Darwin descubrió con sorpresa las diferencias entre los tres cucuves de las tres islas: San Cristóbal, Floreana e Isabela, y no percibió la variedad de los pinzones. El científico británico, John Gould, fue la persona crucial en determinar a los pinzones de Galápagos. Gould fue el taxónomo que recibió las aves colectadas por Charles Darwin y fue él quien enfatizó que los especímenes de los pinzones representaban especies diferentes.

Mucho más tarde, David Lack, quien estuvo en las Islas Galápagos entre 19391940, denominó a los pinzones de Galápa- gos como "Darwin's Finches" (pinzones de Darwin) en su monografía publicada en 1947. Desde entonces, estas avecitas 1levan el nombre de "Darwin". Fueron Peter y Rosemary Grant en sus dos obras Ecology and Evolution of Darwin's Finches y How and why species multiply, quienes, durante un estudio de más de tres décadas (1973 hasta la presente), aclararon cómo y por qué las especies se multiplican.

Un hito en los estudios de la evolución es el libro El Pico del Pinzón de Jonathan Weiner, en el cual demuestra una historia de evolución que ocurre en nuestro tiempo: La evolución no es una cosa del pasado, es un proceso que pasa bajo nuestros propios ojos. Lo que Lack y Bowman, quienes estuvieron en la década de 1950 en las Islas Galápagos, no pudieron descifrar, fue analizado por Peter y Rosemary Grant. Con grandes dosis de paciencia y dedicación ellos midieron miles y miles de picos de pinzones, generación tras generación. Notaron cambios en el tamaño de los picos en una población que estuvo sometida a diferentes condiciones ambientales. De este modo demostraron que el proceso de la selección natural ocurre en una misma generación y que la evolución se da en varias generaciones. Peter y Rose- 
mary Grant (2002) también demuestran cómo el número de las especies de pinzones aumentan con el número de las islas en Galápagos. Hace 3 ó 4 millones de años había una especie de pinzón y cuatro islas; desde hace un millón de años, cinco pinzones y siete islas. El mayor proceso evolutivo de los pinzones ocurrió desde hace un millón de años hasta el presente. En este periodo las islas aumentaron de 7 a 18 y las especies de pinzones de 5 a 15 .

Pero regresemos a Charles Darwin y su sorprendente descubrimiento sobre la variación de los tres cucuves. Observó además al Gavilán de Galápagos (Buteo galapagoensis, Gould 1837) e hizo comentarios sobre el comportamiento de esta ave rapaz que tiene costumbres de un "caracara". Solamente podemos especular sobre qué pasaría por la mente de Charles Darwin si hubiese observado a los pingüinos y a los cormoranes no voladores. Seguramente navegó por entre estas aves cuando el H.M.S. Beagle pasó entre las islas Isabela y Fernandina. Estas especies fueron descritas mucho más tarde (Spheniscus mendiculus, Sundevall 1871 y Phalacrocorax harrisi, Rothschild 1898) y tienen adaptaciones muy especiales a las condiciones de Galápagos. Charles Darwin no vio estas aves y por ende se quedó solamente con el descubrimiento sorprendente de la variación entre los cucuves de tres islas y no se percató sobre la variación de los pingüinos en dos mundos tan distintos como las Galápagos y la Antártida, ni tampoco reflexionó sobre cormoranes que no vuelan.

\section{BIBLIOGRAFÍA}

Bowman, R.I., 1961. Morphological differentiation and adaptation in the Galapagos finches. University of California Publications in Zoology 58:1-302.

Grant, P.R., 1999. Ecology and Evolution of Darwin's Finches. Princeton Univ. Press, Princeton NJ, USA.

Grant, P.R. y B.R. Grant, 2002. Adaptive Radiation of Darwin's Finches. American Scientist 90:130-139.

Grant, P.R. y B.R. Grant, 2008. How and Why Species Multiply. Princeton Univ. Press, Princeton NJ, USA.

Lack, D., 1947. Darwin's Finches. Reissued 1983. Cambridge Univ. Press, Cambridge, UK.

Weiner, J., 1994. The Beak of the Finch. Vintage Books, Random House, Inc., New York, USA. 